\title{
Do microscopic merging models reproduce the observed priority sharing ratio in congestion?
}

\author{
Estelle Chevallier, Ludovic Leclercq * \\ Université de Lyon, ENTPE / INRETS - Laboratoire d'Ingénierie Circulation Transports, Rue Maurice Audin, 69518 Vaulx-en-Velin Cedex, France
}

\section{A R T I C L E I N F O}

\section{Article history:}

Received 16 May 2008

Received in revised form 5 January 2009

Accepted 5 January 2009

Available online $\mathrm{xxxx}$

\section{Keywords:}

Merge

Microscopic merging model

Priority sharing

Congestion

Relaxation

Flow allocation

Gap-acceptance

\begin{abstract}
A B S T R A C T
A classical way to represent vehicle interactions at merges at the microscopic scale is to combine a gap-acceptance model with a car-following algorithm. However, in congested conditions (when a queue spills back on the major road), outputs of such a combination may be irrelevant if anticipatory aspects of vehicle behaviours are disregarded (like in single-level gap-acceptance models). Indeed, the insertion decision outcomes are so closely bound to the car-following algorithm that irrelevant results are produced. On the one hand, the insertion decision choice is sensitive to numerical errors due to the car-following algorithm. On the other hand, the priority sharing process observed in congestion cannot be correctly reproduced because of the constraints imposed by the car-following on the gap-acceptance model. To get over these issues, more sophisticated gap-acceptance algorithms accounting for cooperation and aggressiveness amongst drivers have been recently developed (multi-level gap-acceptance models). Another simpler solution, with fewer parameters, is investigated in this paper. It consists in introducing a relaxation procedure within the car-following rules and proposing a new insertion decision algorithm in order to loosen the links between both model components. This approach will be shown to accurately model the observed flow allocation pattern in congested conditions at an aggregate scale.
\end{abstract}

(c) 2009 Elsevier Ltd. All rights reserved.

\section{Introduction}

Microscopic frameworks seem to be well-suited for capturing the complexity and uncertainty involved in vehicle interactions at merges. They are based on two components: (i) the car-following algorithm which simulates vehicle trajectories on both the minor and the major roads; (ii) the insertion decision model which specifies whether the demand for insertion could be met according to the traffic conditions upstream and downstream of the conflict point.

A topic of increasing concern is the validity of these two microscopic sub-models. Insertion decision models are not straightforward to compare due to their large diversity. The most complex of them simulate mixed strategy between conflicting vehicles with respect to a multi-agent game theory (Kita, 1999; Liu et al., 2007). However, they are rarely implemented within simulation packages and are currently of little practical interest since they contain several behavioural parameters which may be difficult to calibrate from external traffic observations. The other class of insertion decision models, which can be more easily implemented into microsimulation packages, is based on gap-acceptance rules. These rules are usually expressed as a single-level binary choice problem: the outcome of the insertion decision process is assessed only once per time-step, independently of what happened or is going to happen at the merge. This is the basis of what is called in the sequel single-level gap-acceptance models like, for instance, the Pollatschek's model (Pollatschek et al., 2002), MOBIL

\footnotetext{
* Corresponding author. Tel.: +33 472047716; fax: +33 472047712.

E-mail address: leclercq@entpe.fr (L. Leclercq).
} 
(Kesting et al., 2007), CORSIM 1.01 (FHWA, 1996), AIMSUN (Barcelò and Casa, 2002) or VISSIM 4.0 (PTV AG, 2004 ). To the authors' knowledge, all validation studies of those models have been conducted when the major road remains in free-flow regime (uncongested regime). Their results in terms of insertion rate, delay and queue length are usually convincing and agree with the stochastic flow allocation pattern occurring in such a situation. However, the ability of single-level gap-acceptance models in capturing merging behaviours when a congestion spills back on the major road (congested regime) is arguable (Hidas, 2005b). In this case, the flow allocation pattern turns out to be more deterministic. On-field studies reveal that a priority sharing process sets up between vehicles of both incoming roads: instead of giving-way, minor stream vehicles merge together with major stream vehicles in some fixed priority sharing ratio, $\gamma$. This phenomenon has been observed by Cassidy and Ahn (2005) and recently by Bar-Gera and Ahn (submitted for publication) on freeways and by Troutbeck (2002) in an urban context. To model it, recent studies proposed complex gap-acceptance models based on a multi-level decision process (multi-level gap-acceptance-models). These models allow for simulating the anticipatory aspect of cooperation or aggressiveness amongst drivers by assessing the insertion decision outcome within a time-scale longer than the simulation step (Ahmed, 1999; Hidas, 2005a; Choudhury et al., 2007; Toledo et al., 2007). However, such models have numerous parameters that may be difficult to measure and adapt to specific site conditions.

The aim of this paper is to demonstrate that a simpler and more parsimonious approach can be implemented in congested regime to catch the effects of the priority sharing process. Development of such an approach is based on a two-step process. In the first step, classical single-level gap-acceptance models are investigated in details to pinpoint why they fail in reproducing observations. For this, a simple but representative microscopic model of this family is chosen to study the deviations between the simulated inflows and the expected flow allocation pattern. The main flaw of the model seems to be bound to the direct interactions between the car-following algorithm and the insertion decision model. In the second step, new modelling principles are proposed to loosen the dependence between both model components. Rather than complicating the insertion rules (as done in multi-level gap-acceptance models), a new microscopic merging framework is proposed. It consists in: (i) a novel insertion decision algorithm based on a continuous insertion rate function instead of a gap-acceptance process; (ii) a relaxation procedure implemented within the car-following model. With a limited set of parameters, this proposed framework will be shown to reproduce the priority sharing process observed in congested regime.

The first part of this paper will express how the observed flow allocation pattern in congested regime can be formalised for its use as a benchmark in the investigation of single-level gap-acceptance models. It will also outline the tested microscopic simulation tool representative of such a model family. In the second section, the simulated and expected inflows derived from both elements will be compared to highlight why single-level gap-acceptance models fail in reproducing merging behaviours in congested regime. The new proposed microscopic framework will be exposed in the third section before concluding with some remarks and prospective ideas.

\section{Elements of the comparison study}

\subsection{Observed flow allocation pattern}

Fig. 1 presents the configuration of interest where a minor road merges into a major road. In congested regime, several on-field studies revealed that vehicles on both incoming roads attempt to optimize the allocation of the downstream capacity, $\Omega$ (Cassidy and Ahn, 2005; Bar-Gera and Ahn, submitted for publication; Troutbeck, 2002). In case of continuous queuing on the major and minor roads, the inflows crossing the merge from the major road $q_{1}$ and from the minor road $q_{2}$ are related to the priority sharing ratio $\gamma$ by:

$$
\left(q_{1}, q_{2}\right)=\left(\frac{\Omega}{1+\gamma}, \frac{\Omega \gamma}{1+\gamma}\right)
$$

Eq. (1) implies that $q_{2} / q_{1}=\gamma$ whatever the value of $\Omega$. This result will stand as a benchmark in the comparison study of Section 3.

As explained by Troutbeck (2002) in an urban context, this phenomenon is mainly due to: (i) a reduction in the risk associated with the insertion manoeuvre because of lower speeds on the major road; (ii) more uniform headways because of queues on both roads.

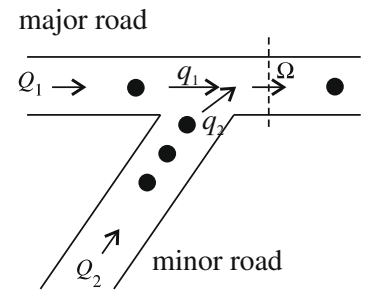

Fig. 1. Notations. 
Note that this observed flow allocation pattern lies at the root of the well-known Daganzo's macroscopic merging model (Daganzo, 1995).

\subsection{Tested microscopic single-level gap-acceptance model}

To identify the limitations and irregularities of single-level gap-acceptance merging models a representative model is tested in Section 3. Its two components - the car-following and the insertion decision algorithms - are described below.

\subsubsection{Car-following algorithm}

2.2.1.1. Algorithm. The car-following algorithm is based on a general formulation of Newell's simplified model (Newell, 2002). It corresponds to the microscopic resolution of the LWR model (Lighthill and Whitham, 1955; Richards, 1956). Details on its derivation can be found in Leclercq et al. (2007a) or Leclercq (2007). The position of vehicle $n$ at time $t+\Delta t, x_{n}^{t+\Delta t}$, is given as the minimum between the position it is willing to reach (demand term) and the position it cannot overpass in congestion due to the downstream vehicle $n-1$ (supply term),

$$
X_{n}^{t+\Delta t}=\min [\underbrace{x_{n}^{t}+u \Delta t}_{\text {demand term }} ; \underbrace{(1-\kappa w \Delta t) x_{n}^{t}+\kappa w \Delta t x_{n-1}^{t}-w \Delta t}_{\text {supply term }}]
$$

where $\Delta t$ is the time-step, $\kappa$ is the jam-density, $\mathrm{u}$ is the free-flow speed and $w$ the wave speed in congested regime. Note that for the reference time-step, $\Delta t=1 /(w \kappa)$, this scheme is free of numerical error (Leclercq, 2007).

The choice of the car-following model is of little impact in the comparison study of Section 3. Indeed, in congested regime, major stream vehicles are closely spaced. Because of insertions from the minor road, spacings may become shorter than the desired ones in equilibrium conditions. In this case, most existing car-following models may be subject to the overreaction effect which is characterised by an underestimation of speeds compared to observations (see Laval and Leclercq (2008) for details). Since complex car-following models are unlikely to produce better results as reported by Brockfeld et al. (2004), it seems relevant to choose a simple model for which the numerical errors can be quantified with respect to the simulation time-step.

2.2.1.2. Input data and parameter values. In the microscopic simulation tool, the congested regime is reproduced by reducing the maximum speed allowed on the downstream link to decrease $\Omega$ compared to the maximum road capacity $q_{m}=u w \kappa /$ $(u+w)$. Condition of continuous queuing on both incoming roads are obtained by setting $Q_{2}=q_{m}$ and $Q_{1}>\Omega(\Omega$ varies). Note that the way vehicles are created at the beginning of each road does not matter since, farther away, headways are constrained by the queuing process.

For simplicity, the parameters of the car-following model are assumed identical for both the major and the minor roads. Their values are chosen to be representative of urban links (Leclercq, 2005): $\kappa=0.18 \mathrm{veh} / \mathrm{m}, u=14 \mathrm{~m} / \mathrm{s}, w=3.47 \mathrm{~m} / \mathrm{s}$. It should be noted that those assumptions do not affect the conclusions of the comparison study of Section 3 .

Simulations are performed on a 2000s-period to catch the within-run variability. A running-in period of 100 s is used. Several simulations with different random number seeds have been conducted to check that the presented results do not depend on the run.

\subsubsection{Insertion decision algorithm}

2.2.2.1. Algorithm. At each simulation time-step, the chosen insertion decision algorithm compares prevailing upstream and downstream traffic conditions on the major road, $S_{u}$ and $S_{d}$, with critical values, $S_{u}^{0}$ and $S_{d}^{0}$, to determine whether a minor stream vehicle can enter the merge. The insertion decision making process is modelled as a binary choice problem with the choice indicator variable $Y$ :

$$
Y(t)= \begin{cases}1 & \text { if } S_{u} \leq S_{u}^{0} \text { and } S_{d} \leq S_{d}^{0} \\ 0 & \text { otherwise }\end{cases}
$$

Two different single-level gap-acceptance models have been tested in the comparison study.

The first one is set by taking $S_{u}$ and $S_{d}$ equal to the distance between the conflict point and the positions of the lag and the lead major stream vehicles at the beginning of the time-step (see $d_{u}$ and $d_{d}$ in Fig. 2a). It is referred as the distance criteria model in the sequel. Since our car-following model implies that all vehicles drive at $\mathrm{u}$ in uncongested regime, this insertion decision model corresponds to most of the existing single-level gap-acceptance models based on a time criteria when the merge is uncongested like in CORSIM 1.01 (FHWA, 1996) and on a distance criteria when it is congested like in VISSIM 4.0 (PTV AG, 2004).

Difficulties in calibrating the critical time or distance values (Brilon et al., 1999) and the need to ensure consistency between the car-following and the lane-changing models have recently motivated the development of decision models formulated in terms of speed difference (Kesting et al., 2007). To account for them in our study, we have tested a second decision model. The upstream criteria $S_{u}$ is equal to the current speed of the lag vehicle, $v_{u 1}$, minus the speed it would have if there 
a

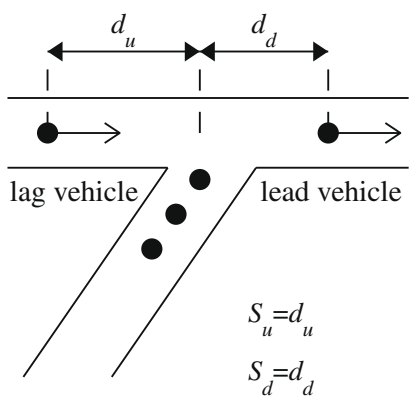

b

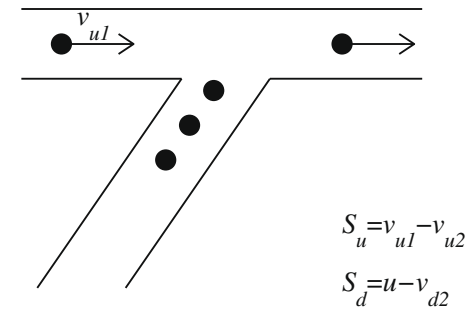

insertion

Fig. 2. Tested insertion decision models: (a) distance criteria and (b) speed differential criteria.

were an insertion, $v_{u 2}$. The downstream criteria $S_{d}$ corresponds to the difference between the minor stream vehicle speed if it enters the merge, $v_{d 2}$, and $u$. This model is depicted in Fig. $2 \mathrm{~b}$ and is called the speed differential criteria model.

When $Y(t)=1$, the time-step is available for insertion. If the minor stream vehicle is expected to arrive at the conflict point within $\Delta t$, it enters the merge and moves to the position calculated according to the car-following algorithm at the end of the simulation time-step.

2.2.2.2. Calibration of the decision model. The critical values $S_{u}^{0}$ and $S_{d}^{0}$ are calibrated to reproduce as closely as possible the expected flow allocation pattern observed in congested regime. They should, therefore, become less and less stringent as the severity of the congestion increases in order to favour insertions of minor stream even if spacing and time-headway between major stream vehicles decrease. However, the interactions between the car-following algorithm and the insertion decision model impose some limit conditions to avoid inconsistent vehicle trajectories:

- the insertion of the minor stream vehicle should not cause the lag vehicle to drive backwards: $S_{u}^{0} \geq 1 / \kappa$ for the distance criteria model and $S_{u}^{0} \leq v_{u 1}$ for the speed differential criteria model;

- the position of the inserting vehicle imposed by the lead vehicle at the end of the time-step should not be upstream of the conflict point: $S_{d}^{0} \geq 1 / \kappa$ for the distance criteria model and $S_{d}^{0} \leq u$ for the speed differential criteria model.

In the sequel, $S_{d}^{0}$ and $S_{u}^{0}$ are set equal to these bound values.

\section{Shortcomings of single-level gap-acceptance models}

Fig. 3 depicts the simulated ratio $q_{2} / q_{1}$ obtained with the tested microscopic single-level gap-acceptance model when $\Omega$ varies, for different values of $\Delta t$ and both kinds of insertion decision models. Results are not relevant with the expected flow allocation pattern which stipulates that the ratio is independent of $\Delta t$ and remain constant whatever the value of $\Omega$. Note that the speed differential model are more consistent, with less dispersion in the simulated ratio when $\Delta t$ or $\Omega$ vary.

The following subsections pinpoint two reasons explaining this inconsistency. They are both related to the interactions between the car-following and the insertion decision algorithms and are inherent to all single-level gap-acceptance models.
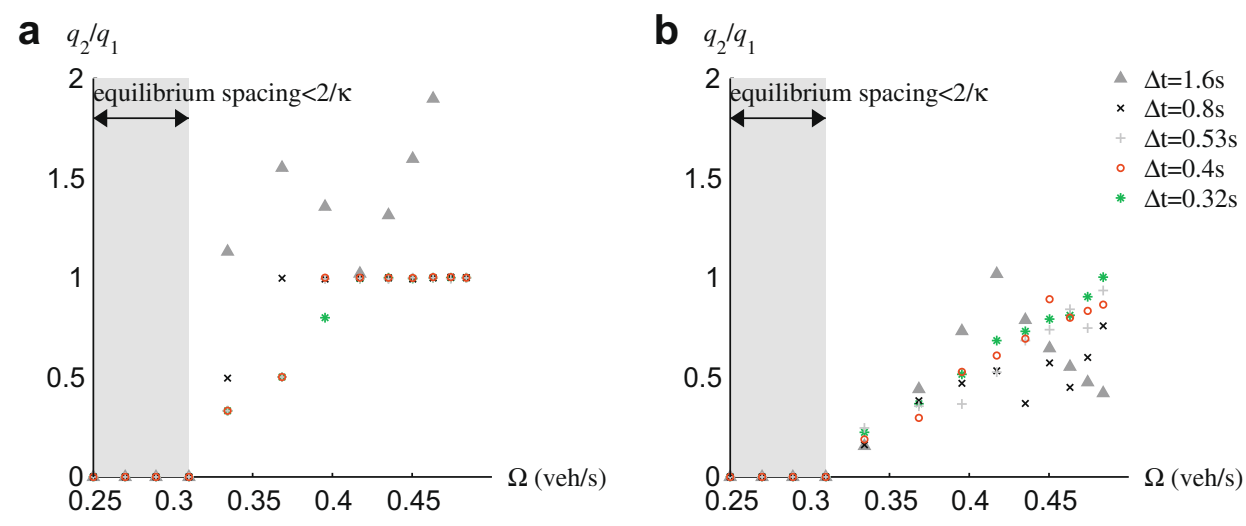

Fig. 3. Variations of $q_{2} / q_{1}$ with $\Delta t$ and $\Omega$ : (a) distance criteria and (b) speed differential criteria. 


\subsection{Errors induced by numerical errors of the discretization method}

In congested regime, most of the car-following algorithms are affected by slight but non-negligible numerical errors as soon as $\Delta t$ exceeds a few tenths of a second. This occurs, for instance: (i) in large-scale applications of microscopic packages when the goal is to reduce the computational costs; (ii) in models in which the reaction time is wrongly accounted for by the time-step, so that $\Delta t$ ranges between $1 \mathrm{~s}$ and $2.2 \mathrm{~s}$ (May, 1990). In these cases, numerical viscosity induces errors in vehicle trajectories. Since the magnitude of the errors is not directly related to $\Delta t$, changes in car-following parameters to correct the model results are unfruitful. Illustration of viscosity errors is given in Fig. 4 which compares four vehicle trajectories computed with two different time-steps. Initially:

- two vehicles $\left(M_{1}\right.$ and $\left.M_{2}\right)$ are created on the major road with a 2.5 s-headway,

- the speed on the major road is $7 \mathrm{~m} / \mathrm{s}$ due to a downstream congestion,

- two minor stream vehicles $\left(m_{1}\right.$ and $\left.m_{2}\right)$ are queuing upstream of the merge since they are artificially prevented to merge until the passing-by of $M_{1}$.

For both time-steps, the first minor stream vehicle, $m_{1}$, enters the merge as soon as $S_{d}^{0}$ is satisfied by $M_{1}$. This insertion forces $M_{2}$ to slow-down. For the reference time-step ( $\Delta t=1.6 \mathrm{~s}$ ), the car-following model (2) is free of numerical errors. Thus, $M_{2}$ completely stops at a $1 / \kappa$-distance from the conflict point during a whole time-step. At the end of this time-step, $m_{1}$ is sufficiently far from the conflict point for $S_{d}^{0}$ to be satisfied. As $S_{u}^{0}$ is still met, $m_{2}$ can enter the merge and $M_{2}$ remains stopped during the next time-step (see Fig. 4a). For the lower time-step, viscosity appears in (2) and causes an anticipation of the trajectory of $M_{2}$ (see Fig. 4b). Therefore, $S_{u}^{0}$ is no more satisfied when $m_{2}$ is ready to enter the merge. As a result, $m_{2}$ should wait whereas $M_{2}$ is allowed to cross the merge Fig. 4 demonstrates that slight errors in the car-following algorithm have a substantial impact on the frequency of the insertion process which is a very cumbersome issue. The sensitivity of the tested single-level model to numerical errors reinforces the conclusions of Hidas (2005b) who analysed the merging abilities of four microscopic traffic flow models in congested regime. In his study, AIMSUN was shown to be very sensitive to the reaction time wrongly taken as the simulation time-step and VISSIM was found to produce unsatisfactory results when $\Delta t$ is longer than $0.5 \mathrm{~s}$.

\subsection{Underestimation of the insertion flow}

Even if $\Delta t$ is short enough to induce negligible errors in vehicle trajectories, single-level gap-acceptance tools still produce inconsistent inflows when traffic states on the major road become highly congested. Indeed, when $\Omega$ decreases, the probability to satisfy insertion decision criteria falls down and so, the number of insertions is underestimated. Reductions in critical values $S_{d}^{0}$ and $S_{u}^{0}$ can only partly overcome this issue since they are constrained by the car-following algorithm requirements as explained in Section 2.2.2. As a result, three other shortcomings affect the simulated inflows as illustrated by the case $\Delta t=1.6 \mathrm{~s}$ (without viscosity) in Fig. 3:

- the ratio $q_{2} / q_{1}$ tends to decrease with $\Omega$ which is inconsistent with the observations made by Cassidy and Ahn (2005) and Troutbeck (2002);

- for a fixed value of $\Omega$, there is no way to force the model to simulate a given observed value of $q_{2} / q_{1}$ as the decision process has no memory;

- when the equilibrium spacing on the major road falls under $2 / \kappa$, no insertion can occur as depicted by the shaded zone in Fig. 3.
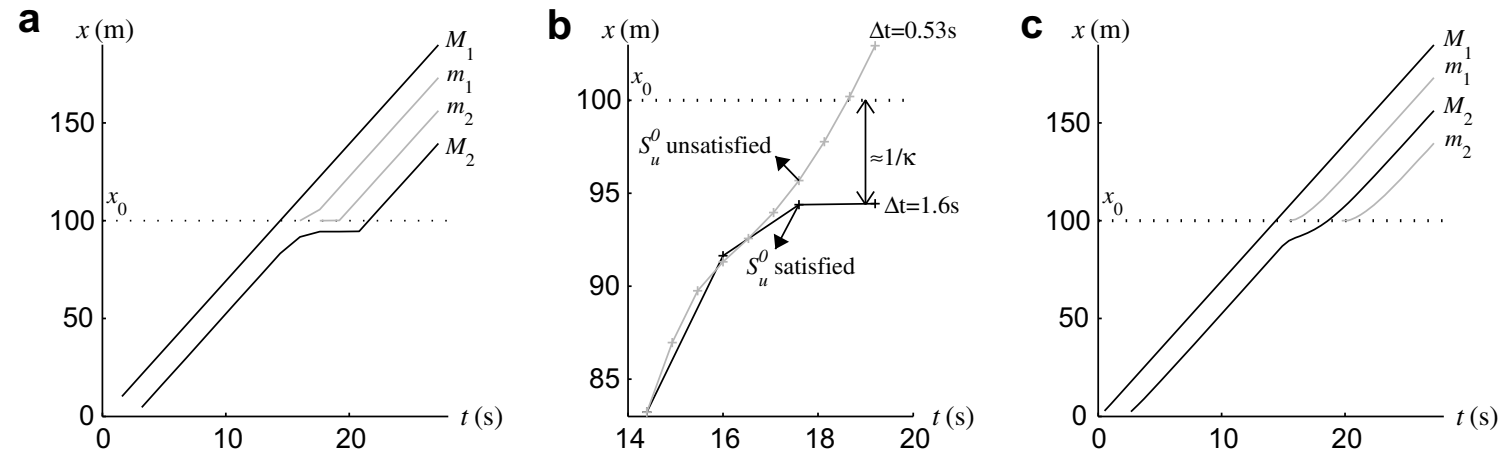

Fig. 4. Effect of numerical errors in single-level gap-acceptance models: (a) $\Delta t=1.6 \mathrm{~s}$ (reference time-step), (b) focus on $M_{2}$ trajectory for both time-step and $(\mathrm{c}) \Delta t=0.53 \mathrm{~s}$. 
As explained in the introduction, the underestimation issue, previously reported by Hidas (2002), has motivated the development of multi-level gap-acceptance models. They enable to relax the links between the insertion decision model and the car-following algorithm by assessing the insertion decision outcome over several time-steps. However, in the light of this section, it appears that efforts remain to be conducted to test the sensitivity of the insertion decision algorithms to numerical errors. Moreover, it should be worth checking the accuracy of those models when traffic conditions on the major road vary abruptly. This is out of the scope of this paper. Instead, we chose to propose a new and simpler approach for overcoming the drawbacks of single-level gap-acceptance models in congested regime by yet keeping the same time-scale for both the insertion decision model and the car-following algorithm.

\section{A new microscopic framework for merges}

The new proposed modelling framework combines an insertion decision model based on a continuous insertion rate function and a relaxation model in order to loosen the dependence between the insertion process and the car-following algorithm.

\subsection{The relaxation model}

A relaxation model aims at allowing non-equilibrium spacings between vehicles and representing how these spacings modify until reaching an equilibrium state (Cohen, 2004; Hidas, 2005a; Skabardonis, 2002). This principle is introduced in our proposed framework through three steps (Laval and Leclercq, 2008):

- the relaxation phase starts as soon as a minor stream vehicle inserts into the major road with a non-equilibrium spacing with its leader and/or its follower. The minor stream vehicle is first considered as a dimensionless moving bottleneck for upstream major stream vehicles;

- this minor stream vehicle (and/or its follower) adapts its speed to increase its spacing with its leader;

- when its spacing reaches an equilibrium state, the whole mass of the minor stream vehicle is transferred onto the major road. This vehicle is then considered as an entire particle moving on the major road according to the classical car-following algorithm.

An interesting contribution of the proposed model (4) is that this procedure is achieved through an extension of Newell's model $(2)$ in case $\Delta t \geqslant 1 /(w \kappa)$ :

$$
x_{n}^{t+\Delta t}=\min \left[x_{n}^{t}+u \Delta t ; x_{n-1}^{t}+v_{n-1}^{t+\Delta t} \Delta t-\frac{\Delta N_{n}^{t+\Delta t}}{k\left(v_{n-1}^{t+\Delta t}\right)}\right]
$$

where $v_{n}^{t+\Delta t}=\frac{x_{n}^{t+\Delta t-x_{n}^{t}}}{\Delta t}$ is the velocity of vehicle $n$ and $k(v)=w \kappa / w+v$ is the equilibrium density corresponding to speed $v . \Delta N_{n}^{t}$ is the ratio between the current spacing in front of vehicle $n$ and the equilibrium spacing associated with the speed of its leader. In the relaxation phase, $\Delta N_{n}^{t}$ is lower than 1 . The time evolution of $\Delta N_{n}^{t}$ is given by:

$$
\left\{\begin{array}{l}
\Delta N_{n}^{t+\Delta t}=\max \left[\Delta N_{n}^{t} ; \min \left[1, \Delta N_{n}^{t} \frac{k\left(v_{n-1}^{t+\Delta t}\right)}{k\left(v_{n-1}^{t}\right)}+\min \left(\frac{\Delta N_{n}^{t}}{w \kappa \Delta t}\left(v_{n-1}^{t+\Delta t}-v_{n-1}^{t}\right)+\varepsilon, v_{n-1}^{t+\Delta t}\right) k\left(v_{n-1}^{t+\Delta t}\right) \Delta t\right]\right] \text { if } \Delta t \geq \frac{S_{0} \Delta N_{n}^{\prime}}{w} \\
\Delta N_{n}^{t+\Delta t}=\max \left[\Delta N_{n}^{t} ; \min \left[1, \Delta N_{n}^{t} \frac{k\left(v_{n-1}^{t}+\Delta t\right)}{k\left(v_{n-1}^{t}\right)}+\min \left(\left(v_{n-1}^{t+\Delta t}-v_{n-1}^{t}\right)+\varepsilon, v_{n-1}^{t+\Delta t}\right) k\left(v_{n-1}^{t+\Delta t}\right) \Delta t\right]\right] \text { otherwise } \\
\Delta N_{n}^{t_{0}}=\left(x_{n-1}^{t_{0}}-x_{0}\right) k\left(v_{n-1}^{t}\right)
\end{array}\right.
$$

Note that if vehicle $n$ is in equilibrium condition, i.e. $\Delta N_{n}^{t}=1$ and if $\Delta t$ is equal to the reference time-step, (4) is fully equivalent to (2).

The proposed model (4) only introduces one additional parameter compared to model (2): the parameter $\varepsilon$. It can be interpreted as the difference in speed the non-equilibrium vehicle is willing to accept with respect to its leader in order to recover an equilibrium spacing. It was found in Leclercq et al. (2007b) that an average value of $\varepsilon$ can provide accurate vehicle trajectories. We took $\varepsilon=0.55 \mathrm{~m} / \mathrm{s}$ in the sequel.

\subsection{The insertion decision model}

\subsubsection{Basic principle}

Thanks to the relaxation model, minor stream vehicles can insert regardless of the car-following requirements. If we keep classical distance or speed differential criteria models, the simulated inflows would still be dependent on the simulation time-step and on the relaxation procedure. To avoid this issue another insertion decision model has been developed in the spirit of the lane-changing model proposed by Laval and Leclercq (2008). It is based on a continuous insertion rate function, $\phi$, corresponding to the maximum inflow, $q_{2}$, allowed from the minor road. It is directly given by the benchmark flow allocation pattern (see Eq. (1)):

$$
\phi(\Omega)=\frac{\Omega \gamma}{1+\Omega}
$$


Then, the continuum process described by the $\phi$-function is discretized into a probabilistic experiment for specifying the outcome of the insertion decision process of each minor stream vehicle. Towards this end, the probability for the arriving minor stream vehicle to enter the merge within the time-step, $p^{t+\Delta t}(\Omega)$, can be computed as follows:

$$
P^{t+\Delta t}(\Omega)=\phi(\Omega) \Delta t
$$

Finally, insertions are executed at time t according to a Bernoulli process with probability of success $p^{t+\Delta t}(\Omega)$. Notice that multiplying $\phi$ by $\Delta t$ in (7) ensures that the insertion decision model is independent of the simulation time-step. Moreover, since $\mathrm{p}^{t+\Delta t}(\Omega)$ is not computed from vehicle trajectories, the insertion rate is no more affected by numerical errors. As a result, the microscopic framework gives the same inflows whatever the simulation time-step.

\subsubsection{Algorithm}

At time-step $t_{0}$, the implementation of the insertion decision model starts by checking that the major road is congested (see Fig. 5). For this, the velocity of the leader is computed from the car-following Eq. (4) and compared to the free-flow speed $u$. Then, the downstream capacity $\Omega$ is calculated from the rates of vehicles $q_{d}(t)$ passing by a downstream location, $x_{d}$, during the last $T$-seconds. The probability for a minor stream vehicle to insert is finally derived from (7).

In the simulations, we arbitrary chose $x_{u}$ and $x_{d}$ at a $20 \mathrm{~m}$-distance upstream and downstream of the conflict point. The value for $T$ should be sufficiently long to smooth the disturbances in flow induced by the relaxation process but short enough to account for dynamics in $\Omega$. To balance these two requirements we took $T=30 \mathrm{~s}$.

\subsection{Results}

Fig. 6a shows the simulated inflows $\left(q_{1}, q_{2}\right)$ for different values of $\Omega$ and $\gamma$. One can see that the ratio $q_{2} / q_{1}$ always tends to $\gamma$ regardless of $\Omega$. Therefore, contrary to single-level gap-acceptance models, $q_{2} / q_{1}$ do not diminish with the severity of congestion and can be controlled through the calibration of $\gamma$.

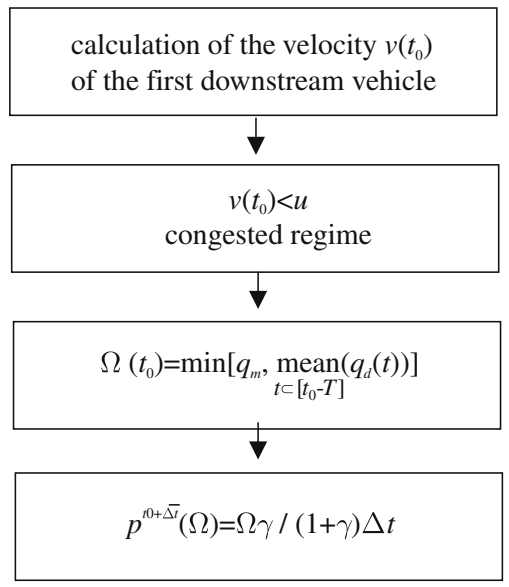

Fig. 5. Overview of the algorithm.

a

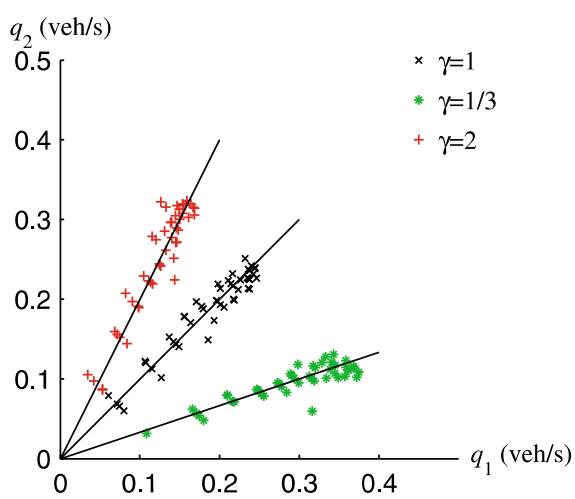

b

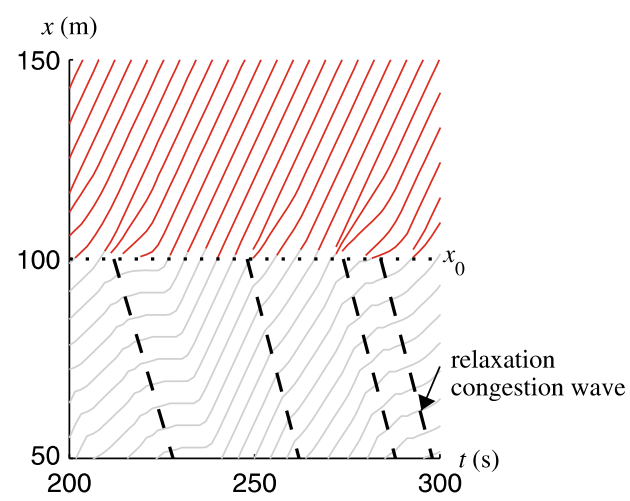

Fig. 6. Results of the proposed microscopic framework: (a) vehicle trajectories on the major road and (b) simulated inflows versus $\Omega$. 
Furthermore, as seen from vehicle trajectories depicted in Fig. 6b, the relaxation model enables the $\phi$-function to be reproduced by the model even if spacings between major stream vehicles are very short. Thus, simulated inserting flows are no longer underestimated like in single-level gap-acceptance models. Minor stream vehicles which accept non-equilibrium spacings with their leader trigger relaxation congestion waves in the major flow. This is illustrated in Fig. $6 \mathrm{~b}$ by dotted lines.

An interesting property of the relaxation model is that even if the maximum outflow at the conflict point can exceed $\Omega$ over very short periods, it is equal to $\Omega$ on average as soon as the downstream link is longer than the distance required by vehicles to relax. The allocation of $\Omega$ between both streams is then specified by the value of the priority sharing ratio $\gamma$.

\section{Conclusion}

Through the comparison of a simple but representative microscopic single-level gap-acceptance model with the observed flow allocation pattern at a merge when the major road is congested, this paper has stressed two fundamental shortcomings of such a model family. Firstly, the insertion rate was shown to be sensitive to the numerical errors appearing in car-following algorithms. Secondly, the observed priority sharing process cannot be controlled and the insertion flow is underestimated as soon as density on the major road reaches one half of the jam-density. Both issues stem from the constraints imposed by the car-following algorithm on the insertion process to avoid inconsistent vehicle trajectories. This explains why multi-level gap-acceptance models, which assess the insertion decision outcome over several time-steps, have been recently developed and shown to provide promising results.

A simpler alternative solution to the issue of merge modelling in congested regime is proposed in this paper. It consists in loosening the interaction between the insertion decision model and the car-following algorithm by: (i) a relaxation procedure implemented within the car-following model; (ii) a new insertion decision model, based on a stochastic algorithm whose mean insertion frequency is independent of the simulation time-step and is fully consistent with the observed priority sharing process. Note that this solution is not based on a detailed description of the cooperation mechanism between the major and the minor stream vehicles but on its macroscopic outcome. The proposed framework gives accurate insertion rates and mimics the average effects of complex merging behaviours such as forced and cooperative merging in a simple way. The only requirement for its implementation is to specify the insertion rate function, $\phi$, representing the mean frequency of the stochastic insertion process. Contrary to multi-level gap-acceptance models that involve a large number of parameters, this framework only requires the calibration of the priority sharing ratio, $\gamma$. This can be done from average flows measured by sensors upstream of the merge or directly by the lane-ratio between incoming roads as recently demonstrated by Bar-Gera and Ahn (submitted for publication).

The proposed framework can account for multiclass traffic by adapting the car-following model (Leclercq and Laval, 2007) and investigating how the insertion rate function can be distributed amongst vehicles. It can be combined with a classical gap-acceptance model implemented in uncongested regime to form a global merging model able to represent all driving behaviours whatever traffic conditions on the major road. Further work is yet needed to check the relevance of the model when the major stream demand varies. Particularly, it should be worth studying how simulated inflows are affected by the convergence time of the Bernoulli process included into the insertion decision model.

\section{References}

Ahmed, K.I., 1999. Modeling driver's acceleration and lane changing behaviour. PhD Dissertation, Department of Civil and Environmental Engineering, MIT. Barcelò, J., Casa, J., 2002. Dynamic network simulation with AIMSUN. In: Proceedings of the International Symposium on Transport Simulation, Yokohama. Bar-Gera, H., Ahn, S., submitted for publication. Empirical macroscopic evaluation of freeway merge-ratio.

Brilon, W., Koenig, R., Troutbeck, R.J., 1999. Useful estimation procedures for critical gaps. Transportation Research Part A 33 (3-4), 161-186.

Brockfeld, E., Kühne, R.D., Wagner, P., 2004. Calibration and validation of microscopic traffic flow models. Transportation Research Record, Journal of the Transportation Research Board 1876, 62-70.

Cassidy, M.J., Ahn, S., 2005. Driver turn-taking behaviour in congested freeway merges. Transportation Research Record, Journal of the Transportation Research Board 1934, 140-147.

Choudhury, C., Ben-Akiva, M.E., Toledo, T., Rao, A., Lee, G., 2007. State dependence in lane changing models. In: Allsop, R.E., Bell, M.G.H., Heydecker, B.G. (Eds.), Proceedings of the 17th International Symposium on Transport Simulation. Elsevier, London, pp. 711-733.

Cohen, S.L., 2004. Application of a relaxation procedure for lane changing in microscopic simulation models. Transportation Research Record, Journal of the Transportation Research Board 1883, 50-58.

Daganzo, C., 1995. The cell transmission model, Part II: network traffic. Transportation Research Part B 29 (2), $79-93$.

FHWA, 1996. CORSIM User Manual-Version 1.01. No. DTFH61-92-Z-00074, Office of Safety and Traffic Operations R\&D, Intelligent Systems and Technology Division, Virginia.

Hidas, P., 2002. Modelling lane changing and merging in microscopic traffic simulation. Transportation Research Part C 10 (5-6), $351-371$.

Hidas, P., 2005a. Modelling vehicle interactions in microscopic simulation of merging and weaving. Transportation Research Part C 13 (1), $37-62$.

Hidas, P., 2005b. Lane changing and merging under congested conditions in traffic simulation models. In: Brebbia, C.A., Wadhwa, L.C. (Eds.), Urban Transport XI - Urban Transport and the Environment in the 21st Century. WIT Press, Southampton, Boston, pp. 79-788.

Kesting, A., Treiber, M., Helbing, D., 2007. MOBIL: general lane-changing model for car-following models. In: Proceedings of the TRB Annual Meeting, Washington, DC.

Kita, H., 1999. A merging-giveway interaction model of cars in a merging section: a game theoretic analysis. Transportation Research Part A 33 (3-4), 305312 .

Laval, J.A., Leclercq, L., 2008. Microscopic modeling of the relaxation phenomenon using a macroscopic lane-changing model. Transportation Research Part B $42(6), 511-522$.

Leclercq, L., 2005. Calibration of flow-density relationships on urban streets. Transportation Research Record, Journal of the Transportation Research Board $1934,226-234$ 
Leclercq, L., 2007. Hybrid approaches to the solutions of the Lighthill-Whitham-Richards model. Transportation Research Part B 41 (7), $701-709$.

Leclercq, L., Laval, J., 2007. A multiclass car-following rule based on the LWR model. In: Traffic and Granular Flow, Paris, France, pp. $735-753$.

Leclercq, L., Laval, J., Chevallier, E., 2007a. The Lagrangian coordinates and what it means for first order traffic flow models. In: Allsop, R.E., Bell, M.G.H., Heydecker, B.G. (Eds.), Proceedings of the 17th International Symposium on Transport Simulation. Elsevier, London, pp. 735-753.

Leclercq, L., Chiabaut, N., Laval, J., Buisson, C., 2007b. Relaxation phenomenon after changing lanes: an experimental validation with the NGSIM dataset. Transportation Research Record, Journal of the Transportation Research Board 1999, 79-85.

Lighthill, M.J., Whitham, J.B., 1955. On kinematic waves II: a theory of traffic flow in long crowded roads. Proceedings of the Royal Society A229, $317-345$.

Liu, H.X., Xin, W., Adam, Z., Ban, X.J., 2007. A game theoretical approach for modelling merging and yielding behaviour at freeway on-ramp sections. In: Allsop, R.E., Bell, M.G.H., Heydecker, B.G. (Eds.). Elsevier, London, pp. 197-211.

May, A.D., 1990. Traffic Flow Fundamentals. Prentice Hall, Englewood Cliffs, NJ.

Newell, G.F., 2002. A simplified car-following theory: a lower-order model. Transportation Research Part B 36 (3), $195-205$.

Pollatschek, M.A., Polus, A., Livneh, M., 2002. A decision model for gap acceptance and capacity at intersections. Transportation Research Part B 36 (7), 649663.

PTV AG, 2004. VISSIM User Manual-Version 4.0, Chapter 6: Traffic/Transit Networks, Karlsruhe.

Richards, P.I., 1956. Shockwaves on the highway. Operation Research 4, 42-51.

Skabardonis, A., 2002. Simulation of freeway weaving areas. Transportation Research Record, Journal of the Transportation Research Board 1802, 115-124.

Toledo, T., Koutsopoulos, H.N., Ben-Akiva, M., 2007. Integrated driving behavior modeling. Transportation Research Part C 15 (2), $96-112$.

Troutbeck, R.J., 2002. The performance of uncontrolled merges using a limited priority process. In: Taylor, M. (Ed.), Proceedings of the 15th International Symposium on Transportation and Traffic Theory. Pergamon, Amsterdam, pp. 463-482. 